\title{
Terminal value problem for a generalized fractional ordinary differential equation
}

\author{
Can $\mathrm{Li}^{1}$, Min-Min $\mathrm{Li}^{1}$, and Han Zhou ${ }^{2}$ \\ ${ }^{1}$ Xi'an University of Technology \\ ${ }^{2}$ Hebei University of Technology
}

July 4, 2020

\begin{abstract}
The present work is concerned with the well-posedness and efficient numerical algorithm for a terminal value problem with a generalized Caputo fractional derivative. We investigate the existence and uniqueness of the solution of the terminal value problem, and consider the continuous dependence of the solutions on the given data. To illustrate our theoretical results, we present a one step algorithm for solving the considered problems. Some numerical examples are shown to illustrate the theoretical results and the efficiency of the numerical method.
\end{abstract}

\section{Hosted file}

liliT-v3.pdf available at https://authorea.com/users/339765/articles/465913-terminal-valueproblem-for-a-generalized-fractional-ordinary-differential-equation 